\title{
ESTIMATING OF BIRTH WEIGHT USING PLACENTAL CHARACTERISTICS IN THE PRESENCE OF MULTICOLLINEARITY
}

\author{
Cem TIRINK ${ }^{1 *}$
}

${ }^{1}$ Igdir University, Faculty of Agriculture, Department of Animal Science, Igdir, Turkey

Received: September 01, 2020; Accepted: September 10, 2020; Published: October 01, 2020

\begin{abstract}
In this study, it was aimed to compare the performance of proposed estimators in the presence of multicollinearity that will be used in regression analysis as an alternative to Least Squares. Birth weight was estimated by using placental features such as sex, placental efficiency, total cotyledon numbers, large cotyledon weight, medium cotyledon weight, small cotyledon weight, large cotyledon number, medium cotyledon number, small cotyledon number, large cotyledon width, medium cotyledon width, small cotyledon width, large cotyledon length, medium cotyledon length, small cotyledon length, large cotyledon depth, medium cotyledon depth, small cotyledon depth for Bafra sheep breed. In the presence of multicollinearity, more reliable models can be obtained by using some estimator. The performances of the Ridge and Liu estimators, which are suggested methods for this situation, were compared. MSE, RMSE, rRMSE, MAPE, $\mathrm{R}^{2}$, and AIC were used as model comparison criteria. As a result of, in the presence of multicollinearity; Liu estimator is recommended as an alternative method to Least Squares.
\end{abstract}

Keywords: Least squares, Ridge estimator, Liu estimator, Multicollinearity, Placental characteristics

*Corresponding author: Igdir University, Faculty of Agriculture, Department of Animal Science, Igdir, Turkey

E mail: cem.tirink@gmail.com (C. TIRINK)

Cem TIRINK (iD) https://orcid.org/0000-0001-6902-5837

Cite as: Tirınk C. 2020. Estimating of birth weight using placental characteristics in the presence of multicollinearity. BSJ Eng Sci, 3(4): 138-145.

\section{Introduction}

The main purpose of animal breeding is to genetically improve populations of livestock and thus produce more efficient production from future populations (Onder and Abaci, 2015; Sen et al., 2019). In general, newborn lambs with higher birth weight are considered as selection criteria that have higher birth weight are selected as breeding material for the future population (Sen et al., 2019).

Reproductive parameters have major effects in small ruminant breeding. In particular, the success of the gestation period effect the health of the newborn lamb (Brzozowska et al., 2020). Postnatal mortality of offspring in sheep breeding depends on placental features (Dwyer et al., 2005). Many studies showed that the placental features have positive and strong correlations between birth weight for newborn lambs (Echternkamp, 1993; Konyali et al., 2007; Alkass et al., 2013; Ozyurek, 2019; Osgerby et al., 2003; Dwyer et al., 2005; Sen et al., 2013; Sen and Onder, 2016). Placental features are more important indicators for the birth weight in sheep and goat breeding (Ozyurek and Turkyilmaz, 2020). 
Morphometric parameters of the placenta affect fetal growth and thus, placental size play vital roles for the birth weight and detecting the postnatal viability (Sen et al., 2013; Brzozowska et al., 2020).

Placental features give much information for the birth weight, postnatal viability. Researches using many properties are the subject of multivariate statistics. One of the multivariate statistical methods used to reveal the relationships between placental morphological features and birth weight of animals is regression analysis. In multivariate statistical modeling, regression analysis is a process to estimate the relationship between explanatory variables and response variable (Ari and Önder, 2013). Many methods are used to estimate the response variable and the most common of which is the Least Squares (LS) method (Uckardes et al., 2012). LS method requires some assumptions to make an effective model estimation. When in the presence of multicollinearity between explanatory variables is provided from these assumptions, alternative methods such as Ridge estimator, Liu estimator are proposed (Hoerl and Kennard, 1970; Liu, 1993).

The aim of this study, to compare the performance of proposed estimators in the presence of multicollinearity that will be used in regression analysis as an alternative to Least Squares using some placental characteristics in Bafra sheep.

\section{Material and Method}

This study was carried out on 40 Bafra sheep kept on Ondokuz Mayis University research farm unit in Samsun Province of Turkey. For this aim, some placental measurements such as sex (S), placental efficiency (PE), total cotyledon numbers (TCN), large cotyledon weight (LCW), medium cotyledon weight (MCW), small cotyledon weight (SCW), large cotyledon number (LCN), medium cotyledon number (MCN), small cotyledon number (SCN), large cotyledon width $\left(\mathrm{LCW}_{\mathrm{i}}\right)$, medium cotyledon width $\left(\mathrm{MCW}_{\mathrm{i}}\right)$, small cotyledon width $\left(\mathrm{SCW}_{\mathrm{i}}\right)$, large cotyledon length (LCL), medium cotyledon length (MCL), small cotyledon length (SCL), large cotyledon depth ( $\mathrm{LCD}_{\mathrm{e}}$ ), medium cotyledon depth (MCDe), small cotyledon depth (SCDe) were used as explanatory variables. The birth weight of lamb is a response variable. All statistical analyses were performed using the RStudio software ( $R$ Core Team, 2020). The "stats", "lmridge", "liureg" packages were used to perform for LS method, Ridge estimator and Liu estimator, respectively (R Core Team, 2019; Imdad and Aslam, 2018a; Imdad and Aslam, $2018 \mathrm{~b})$. To perform the model selection criteria was used to "ehaGoF" package (Eyduran, 2019).

In the matrix form of multiple regression is:

$Y=X \beta+\varepsilon$

where; $\mathrm{Y}$ is a response variable, $\mathrm{X}$ is a matrix for the explanatory variables and $\beta$ is a vector for regression coefficients and $\varepsilon$ is an error term. The most common method used to estimate the model in regression analysis is the LS method. The main purpose of the LS minimizes the sum of squares of error terms (Kutner et al., 2004).

$\hat{\beta}_{L S}=\operatorname{argmin} \sum\left(y_{i}-\hat{y}_{i}\right)^{2} \mathrm{i}=1,2, \ldots, \mathrm{n}$

Some assumptions need to be provided to estimate the optimum model with LS. The first assumption is that the regression model must be linear. The second assumption is that the value of the expected error for the regression model must be zero. Further, the variance of the errors must be constant and the errors must be independent (Sarstedt and Mooi, 2014). Besides these assumptions, there should not be a linear relationship (multicollinearity) between the explanatory variables (Tirink et al., 2020). In the presence of multicollinearity, the results of the model to be obtained will not reliable (Cankaya et al. 2019).

There are many methods for determining multicollineartiy. One of them is the determination of multicollinearity with Variance Inflation Factor (VIF) value. When the VIF value is greater than 10 , it can be mentioned that there is multicollinearity between the explanatory variables (Albayrak, 2005; Topal et al., 2010).

$V I F=c_{i j}=\frac{1}{1-R_{j}^{2}}$

\subsection{Ridge Estimator}

The ridge estimator, whose main purpose is to obtain more reliable models by eliminating the multicollinearity between the explanatory variables, was proposed by Hoerl and Kennard (1970). In the case of multicollinearity between the explanatory variables, variance and covariances will increase in the $X^{\prime} X$ matrix. (Vupa and Gurunlu Alma, 2008; Uckardes et al., 2012). To reduce the variance and covariances in the $\mathrm{X}^{\prime} \mathrm{X}$ matrix, the bias coefficient $k$ is added to the diagonal elements of the matrix (Tirink et al., 2020). $k$ should be between 0 and 1 . If the bias coefficient of $\mathrm{k}$ is zero, regression parameter estimation is the same as LS (Uckardes et al., 2012). Regression parameter estimation as a matrix notation is given below with the ridge estimator.

$\hat{\beta}_{\text {Ridge }}=\left(X^{\prime} X+k I\right)^{-1} X^{\prime} Y$

It is important to calculate optimum $k$ value. Many researchers suggested a lot of method for calculating the optimum $k$ value. The equation is given below that proposed by Kurtulus (2001) for calculating the optimum bias coefficient of $k$ value based on eigenvalue:

$k \leq \frac{\lambda_{\max }-100 \lambda_{\min }}{99}, k \neq 0$ 


\subsection{Liu Estimator}

The Liu estimator, whose main purpose is to eliminate the multicollinearity between explanatory variables. Liu estimator was proposed by Liu (1993). To eliminate the multicollinearity, the Liu estimator was proposed by combining Stein and Ridge estimator (Alpu and Samkar, 2010). Regression parameter estimation as a matrix notation is given below with the Liu estimator.

$\hat{\beta}_{L i u}=\left(X^{\prime} X+I\right)^{-1}\left(X^{\prime} X+d I\right) \hat{\beta}_{L S}$

$d$ is a biasing parameter used to overcome the multicollinearity. $d$ parameter should be between 0 and 1 . $\hat{\beta}_{\text {Liu }}$ is a linear function of biasing parameter of $\mathrm{d}$ so that to calculate $\mathrm{d}$ is easier than $k$ (Alpu and Samkar, 2010). The equation is given below that for calculating the optimum biasing parameter of $d$ based on the matrix of eigenvectors $(Q)$ (Alpu and Samkar, 2010):

$\hat{d}=1-\hat{\sigma}^{2}\left[\frac{\sum_{i=1}^{p} \frac{1}{\lambda_{i}\left(\lambda_{i}+1\right)}}{\sum_{i=1}^{p} \frac{\hat{\alpha}_{i}^{2}}{\left(\lambda_{i}+1\right)^{2}}}\right], \quad \hat{\alpha}=Q^{\prime} \hat{\beta}_{L S}$

\subsection{Model Selection Criteria}

Mean square error (MSE), root mean square error (RMSE), relative root mean square error (rRMSE), mean absolute percentage error (MAPE), determination of coefficient $\left(\mathrm{R}^{2}\right)$ and Akaike information criteria (AIC) were used as a model selection criteria as To measure the model accuracy of estimators used model comparison criteria as given below.

$M S E=\frac{1}{n} \sum_{i=1}^{n}\left(Y_{i}-\hat{Y}_{i}\right)^{2}$

$R M S E=\sqrt{\frac{1}{n} \sum_{i=1}^{n}\left(Y_{i}-\hat{Y}_{i}\right)^{2}}$

$r R M S E=\frac{\sqrt{\frac{1}{n} \sum_{i=1}^{n}\left(Y_{i}-\hat{Y}_{i}\right)^{2}}}{\bar{y}} * 100$
$M A P E=\frac{1}{n} \sum_{i=1}^{n}\left|\frac{y_{i}-\hat{y}_{i}}{y_{i}}\right| * 100$

$R^{2}=\left[1-\frac{\sum_{i=1}^{n}\left(y_{i}-\hat{y}_{i}\right)^{2}}{\sum_{i=1}^{n}\left(y_{i}-\bar{y}\right)^{2}}\right]$

$A I C=n * \ln \left[\frac{1}{n} \sum_{i=1}^{n}\left(y_{i}-\hat{y}_{i}\right)^{2}\right]+2 p$

Within the scope of model selection criteria, the lowest RMSE, MAPE, AIC values and the highest $\mathrm{R}^{2}$ values were considered for the criteria used in the selection of the best model. (Tatliyer, 2020).

\subsection{Ethical Consideration}

The experiment design was approved by the Local Animal Care and Ethics Committee of Ondokuz Mayis University with an approval number of 2018-31.

\section{Results and Discussion}

In this study, different estimators that are in the presence of multicollinearity were used to estimate the birth weight of Bafra sheep. Descriptive statistics for response and explanatory variables are in Table 1 and Table 2 and correlations among them was given in Figure 1.

\subsection{Results of Least Squares}

Regression coefficients, its standard error, test statistics and significance level obtained from the LS method are given in Table 3. According to the Table 3, intercept, S, SCW, MCL and LCDe were determined to be statistically significant $(\mathrm{p}<0.05)$.

\subsection{Results of Ridge Estimator}

VIF values for each biasing parameter of $\mathrm{k}$ were given in Table 5. In Table 5, the optimum $\mathrm{k}$ value was determined as 0.013 . Regression coefficients, its standard error, test statistics and significance level obtained from the ridge estimator were given in Table 4. According to the Table 4, $\mathrm{S}$ and SCW were determined to be statistically significant $(\mathrm{p}<0.05)$.

\subsection{Results of Liu Estimator}

Regression coefficients, its standard error, test statistics and significance level obtained from the ridge estimator were given in Table 6. According to the Table 6, S, SCW and LCDe were determined to be statistically significant $(\mathrm{p}<0.05)$.

Table 1. Descriptive statistics for explanatory variables

\begin{tabular}{llcccc}
\hline \multirow{3}{*}{ Male } & & $\mathrm{n}$ & Median & Minimum & \multicolumn{2}{c}{ Maximum } \\
& LCN & & 9 & 1 & 39 \\
& MCN & 22 & 22 & 5 & 38 \\
\hline \multirow{3}{*}{ Female } & SCN & & 18 & 4 & 66 \\
& LCN & & 8 & 18 & 21.50 \\
& MCN & 18 & 0 & 51 & 36 \\
\hline
\end{tabular}


Black Sea Journal of Engineering and Science

Table 2. Descriptive statistics for response and explanatory variables

\begin{tabular}{|c|c|c|c|c|c|}
\hline & & $\mathrm{n}$ & Mean \pm Standard Deviation & Minimum & Maximum \\
\hline \multirow{15}{*}{ Male } & BW & \multirow{15}{*}{22} & $5.19 \pm 0.72$ & 3.50 & 6.53 \\
\hline & PE & & $240.99 \pm 104.24$ & 2.32 & 505.71 \\
\hline & TCN & & $53.55 \pm 17.79$ & 26.00 & 89.00 \\
\hline & LCW & & $28.36 \pm 23.01$ & 6.94 & 100.00 \\
\hline & MCW & & $30.57 \pm 12.38$ & 10.19 & 59.00 \\
\hline & SCW & & $15.43 \pm 9.79$ & 1.00 & 34.00 \\
\hline & $\mathrm{LCW}_{\mathrm{i}}$ & & $28.18 \pm 7.75$ & 15.20 & 47.08 \\
\hline & $\mathrm{MCW}_{\mathrm{i}}$ & & $21.9 \pm 4.16$ & 16.40 & 30.22 \\
\hline & $\mathrm{SCW}_{\mathrm{i}}$ & & $15.01 \pm 3.89$ & 9.40 & 23.36 \\
\hline & LCL & & $39.34 \pm 9.04$ & 26.60 & 61.24 \\
\hline & MCL & & $28.4 \pm 4.86$ & 21.00 & 37.20 \\
\hline & SCL & & $19.44 \pm 3.58$ & 14.80 & 27.56 \\
\hline & $\mathrm{LCD}_{\mathrm{e}}$ & & $5.34 \pm 1.51$ & 3.00 & 9.88 \\
\hline & $\mathrm{MCD}_{\mathrm{e}}$ & & $4.85 \pm 1.61$ & 3.00 & 9.81 \\
\hline & $\mathrm{SCD}_{\mathrm{e}}$ & & $3.58 \pm 1.25$ & 2.10 & 6.74 \\
\hline \multirow{15}{*}{ Female } & BW & \multirow{15}{*}{18} & $4.23 \pm 0.57$ & 3.00 & 5.25 \\
\hline & $\mathrm{PE}$ & & $249.82 \pm 93.07$ & 3.25 & 418.12 \\
\hline & TCN & & $49.94 \pm 20.52$ & 15.00 & 74.00 \\
\hline & LCW & & $28.64 \pm 16.52$ & 0.00 & 64.26 \\
\hline & MCW & & $28.76 \pm 12.16$ & 7.34 & 49.00 \\
\hline & SCW & & $20.68 \pm 15.54$ & 4.00 & 70.13 \\
\hline & $\mathrm{LCW}_{\mathrm{i}}$ & & $26.84 \pm 7.93$ & 0.00 & 37.06 \\
\hline & $\mathrm{MCW}_{\mathrm{i}}$ & & $22.81 \pm 4.34$ & 16.57 & 31.44 \\
\hline & $\mathrm{SCW}_{\mathrm{i}}$ & & $16.06 \pm 3.39$ & 10.30 & 22.78 \\
\hline & LCL & & $36.41 \pm 11.67$ & 0.00 & 55.68 \\
\hline & MCL & & $28.87 \pm 5.15$ & 22.72 & 36.87 \\
\hline & SCL & & $20.84 \pm 3.89$ & 15.19 & 26.10 \\
\hline & $\mathrm{LCD}_{\mathrm{e}}$ & & $5.01 \pm 1.79$ & 0.00 & 7.71 \\
\hline & $\mathrm{MCD}_{\mathrm{e}}$ & & $5.04 \pm 1.73$ & 2.75 & 9.85 \\
\hline & $\mathrm{SCD}_{\mathrm{e}}$ & & $4.43 \pm 1.76$ & 2.18 & 8.60 \\
\hline
\end{tabular}

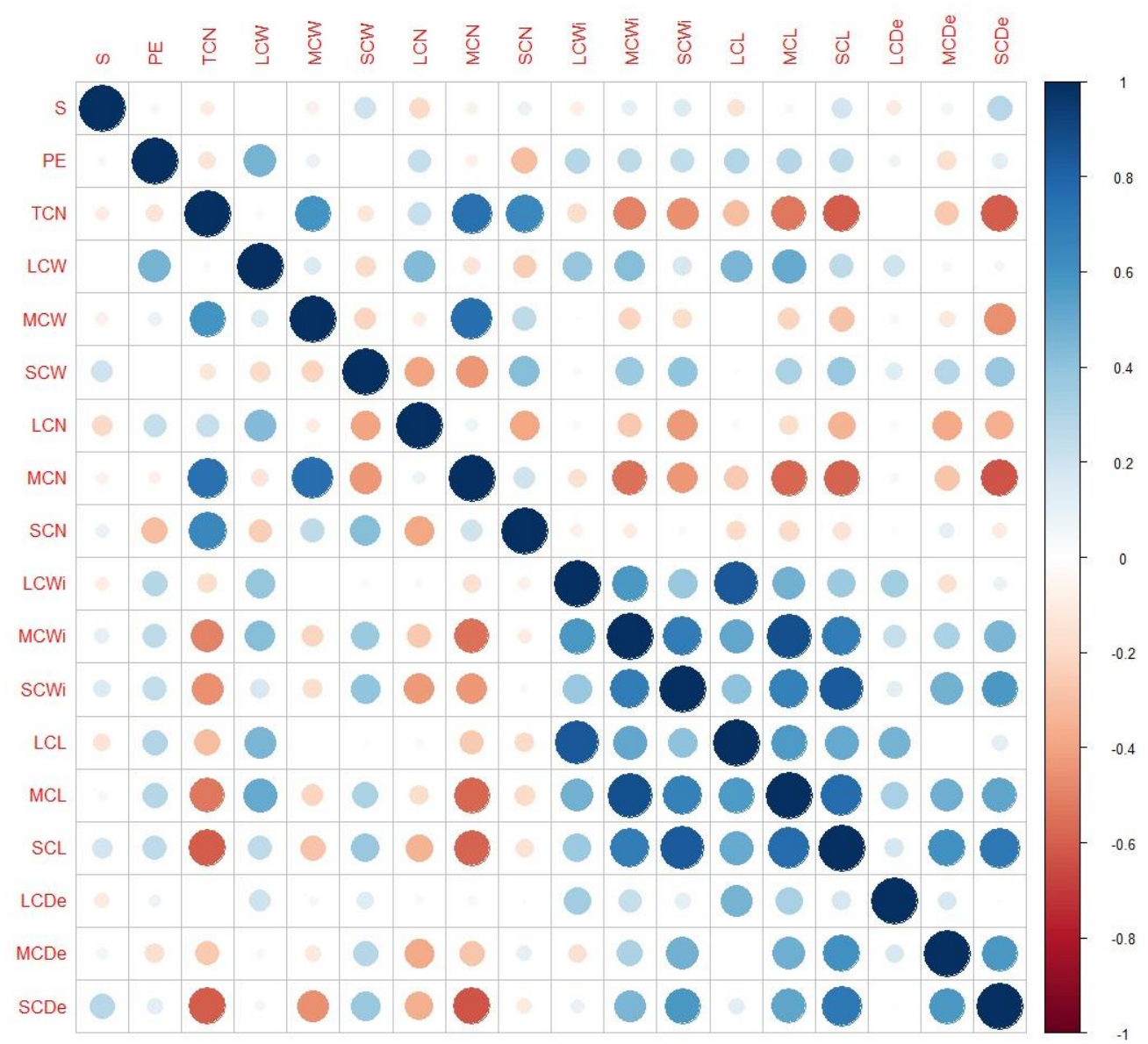

Figure 1. Correlation coefficients for explanatory variables. 
Black Sea Journal of Engineering and Science

Table 3. Results of LS method

\begin{tabular}{lcccc}
\hline & Estimate & Standard Error & $t$-value & p-value \\
\hline (Intercept) & 7.16 & 1.57 & 4.56 & 0.00 \\
S & -0.98 & 0.24 & -4.14 & 0.00 \\
PE & 0.00 & 0.00 & -1.28 & 0.21 \\
CN & 0.06 & 0.04 & 1.50 & 0.15 \\
LCW & 0.02 & 0.01 & 1.75 & 0.09 \\
MCW & -0.01 & 0.02 & -0.80 & 0.43 \\
SCW & 0.03 & 0.01 & 2.43 & 0.02 \\
LCN & -0.06 & 0.05 & -1.18 & 0.25 \\
MCN & -0.02 & 0.04 & -0.61 & 0.55 \\
SCN & -0.08 & 0.05 & -1.63 & 0.12 \\
LCW & 0.00 & 0.04 & -0.01 & 0.99 \\
MCW & 0.06 & 0.07 & 0.84 & 0.41 \\
SCW & 0.01 & 0.06 & 0.17 & 0.86 \\
LCL & 0.02 & 0.03 & 0.60 & 0.55 \\
MCL & -0.12 & 0.06 & -1.89 & 0.07 \\
SCL & 0.00 & 0.09 & -0.02 & 0.98 \\
LCD & -0.18 & 0.08 & -2.19 & 0.04 \\
MCD & 0.10 & 0.13 & 0.81 & 0.43 \\
SCDe & -0.03 & 0.13 & -0.19 & 0.85 \\
\hline
\end{tabular}

Table 4. Results of Ridge estimator

\begin{tabular}{|c|c|c|c|c|}
\hline & Estimate & Standard Error & $t$-value & $\mathrm{p}$-value \\
\hline Intercept & 7.100 & 249.908 & 0.486 & 0.632 \\
\hline S & -0.985 & 0.698 & -4.439 & 0.000 \\
\hline PE & -0.002 & 0.795 & -1.307 & 0.204 \\
\hline $\mathrm{CN}$ & 0.025 & 1.941 & 1.547 & 0.136 \\
\hline LCW & 0.015 & 1.129 & 1.718 & 0.100 \\
\hline MCW & -0.008 & 1.173 & -0.503 & 0.620 \\
\hline SCW & 0.027 & 0.946 & 2.312 & 0.030 \\
\hline LCN & -0.016 & 1.265 & -0.643 & 0.527 \\
\hline MCN & 0.003 & 1.568 & 0.138 & 0.892 \\
\hline SCN & -0.032 & 1.492 & -1.720 & 0.099 \\
\hline $\mathrm{LCW}_{\mathrm{i}}$ & 0.002 & 1.414 & 0.052 & 0.959 \\
\hline $\mathrm{MCW}_{\mathrm{i}}$ & 0.037 & 1.506 & 0.652 & 0.521 \\
\hline $\mathrm{SCW}_{\mathrm{i}}$ & 0.028 & 1.193 & 0.536 & 0.598 \\
\hline LCL & 0.011 & 1.526 & 0.443 & 0.662 \\
\hline MCL & -0.094 & 1.625 & -1.788 & 0.087 \\
\hline SCL & -0.008 & 1.642 & -0.117 & 0.908 \\
\hline LCDe & -0.151 & 0.752 & -2.040 & 0.053 \\
\hline $\mathrm{MCD}_{\mathrm{e}}$ & 0.067 & 1.153 & 0.594 & 0.558 \\
\hline $\mathrm{SCD}_{\mathrm{e}}$ & -0.033 & 1.124 & -0.278 & 0.783 \\
\hline
\end{tabular}




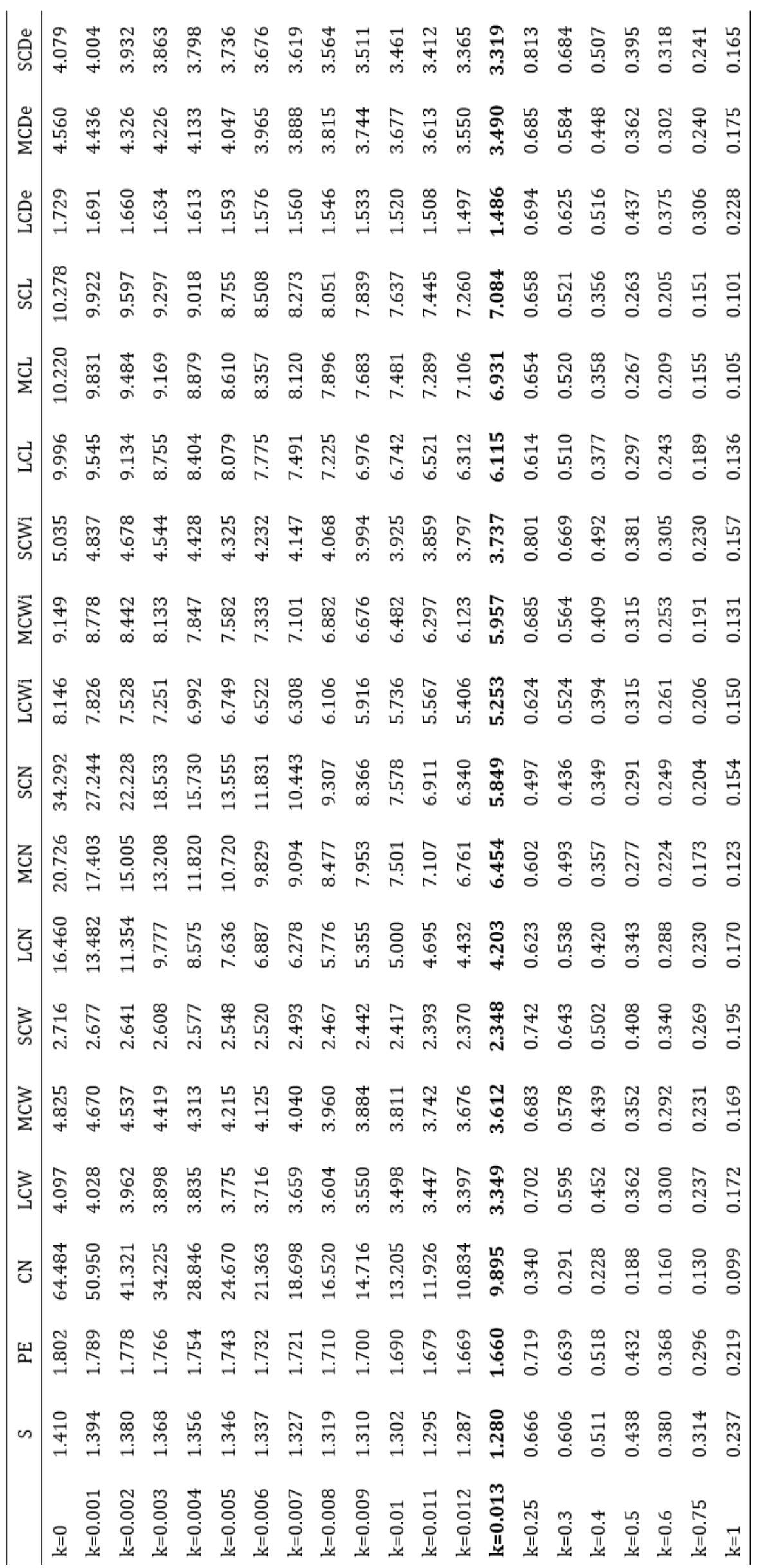


Table 6. Results of Liu estimator

\begin{tabular}{lcccc}
\hline & Estimate & Standard Error & t-value & p-value \\
\hline Intercept & $7.07 \mathrm{E}+00$ & $4.37 \mathrm{E}+00$ & 1.618 & 0.1056 \\
S & $-9.09 \mathrm{E}-01$ & $2.14 \mathrm{E}-01$ & -4.252 & $2.12 \mathrm{E}-05$ \\
PE & $-1.75 \mathrm{E}-03$ & $1.33 \mathrm{E}-03$ & -1.309 & 0.1906 \\
CN & $6.46 \mathrm{E}-02$ & $4.11 \mathrm{E}-02$ & 1.575 & 0.1153 \\
LCW & $1.70 \mathrm{E}-02$ & $9.81 \mathrm{E}-03$ & 1.733 & 0.0831 \\
MCW & $-1.37 \mathrm{E}-02$ & $1.76 \mathrm{E}-02$ & 0.4383 \\
SCW & $3.07 \mathrm{E}-02$ & $1.26 \mathrm{E}-02$ & -0.775 & 0.0145 \\
LCN & $-5.84 \mathrm{E}-02$ & $4.82 \mathrm{E}-02$ & 2.444 & 0.2261 \\
MCN & $-2.58 \mathrm{E}-02$ & $3.78 \mathrm{E}-02$ & -1.211 & 0.4942 \\
SCN & $-7.59 \mathrm{E}-02$ & $4.46 \mathrm{E}-02$ & -0.684 & 0.0889 \\
LCW & $5.49 \mathrm{E}-05$ & $3.56 \mathrm{E}-02$ & -1.701 & 0.9988 \\
MCW & $5.88 \mathrm{E}-02$ & $6.95 \mathrm{E}-02$ & 0.002 & 0.3976 \\
SCW & $1.26 \mathrm{E}-02$ & $5.93 \mathrm{E}-02$ & 0.846 & 0.8314 \\
LCL & $1.94 \mathrm{E}-02$ & $2.97 \mathrm{E}-02$ & 0.213 & 0.5141 \\
MCL & $-1.21 \mathrm{E}-01$ & $6.25 \mathrm{E}-02$ & 0.653 & 0.0539 \\
SCL & $-6.35 \mathrm{E}-03$ & $8.22 \mathrm{E}-02$ & -1.927 & 0.9384 \\
LCD & $-1.76 \mathrm{E}-01$ & $7.81 \mathrm{E}-02$ & -0.077 & 0.0245 \\
MCD & $1.09 \mathrm{E}-01$ & $1.23 \mathrm{E}-01$ & -2.249 & 0.374 \\
SCD & $-2.94 \mathrm{E}-02$ & $1.25 \mathrm{E}-01$ & 0.889 & 0.8133 \\
\hline
\end{tabular}

\subsection{Comparison of the Estimators}

If the assumptions are not provided in the model estimates made with LS, the reliability of the model estimation decreases. In this study, the Ridge and Liu estimator, which is one of the proposed estimators, was used as an alternative method to the LS in cases where the multicollinearity assumption, which is one of the assumptions, was not provided. MSE, RMSE, rRMSE, MAPE, $\mathrm{R}^{2}$, and AIC were used as model comparison criteria. According to the model comparison criteria used in Table 7, it is possible to interpret the best model for the lowest MSE, RMSE, rRMSE, MAPE, $\mathrm{R}^{2}$, and AIC and the highest $\mathrm{R}^{2}$ value (Tatliyer, 2020). In table 7 examined, Liu estimator, used as an alternative estimator to LS, has the lowest MSE, RMSE, rRMSE, MAPE and AIC and the highest $\mathrm{R}^{2}$ value. Tirink et al. (2019) were determined similar results.

Table 7. Model comparison criteria

\begin{tabular}{lccc}
\hline Model Comparison Criteria & Least Squares & Ridge Estimator & Liu Estimator \\
\hline Mean square error (MSE) & 0.205 & 0.214 & 0.206 \\
Root mean square error (RMSE) & 0.453 & 0.463 & 0.454 \\
Relative root mean square error (rRMSE) & 9.51 & 9.726 & 9.53 \\
Mean absolute percentage error (MAPE) & 7.522 & 7.785 & 7.508 \\
Coefficient of determination (R $\left.{ }^{2}\right)$ & 0.679 & 0.664 & 0.677 \\
Akaike's information Criterion (AIC) & -59.398 & -57.602 & -59.236 \\
\hline
\end{tabular}

\section{Conclusion}

In the evaluation of the data for agricultural studies according to cause and effect relationships, in the case of multicollinearity between the explanatory variables, Ridge and Liu estimators were used as an alternative to LS. For this purpose, it was aimed to estimate birth weight by using placental features in Bafra sheep. The results showed that Liu estimator is more reliable model estimation than LS and Ridge estimator. On account of in case of the multicollinearity, the Liu estimator is an alternative to LS.

\section{Conflict of interest}

The author declared that there is no conflict of interest.

\section{References}

Albayrak AS. 2005. An alternative bias estimation technique and an application of the least-squares technique in multiple 
linear connections. Zonguldak Karaelmas Univ J Soc Sci, 1: 105-126.

Alkass JE, Merkhan KY, Hamo RAH. 2013. Placental traits and their relation with birth weight in Meriz and Black goats. Sci J Anim Sci, 2: 168-172.

Alpu 0, Samkar H. 2010. Liu estimator based on an m estimator. Turkiye Klinikleri J Biostat, 2 (2): 49-53.

Ari A, Onder H. 2013. Regression models used for different data structures. Anadolu J Agr Sci, 28 (3): 168-174.

Brzozowska A, Wojtasiak N, Błaszczyk B, Stankiewicz T, Wieczorek-Dąbrowska M, Udała J. 2020. The effects of nongenetic factors on themorphometric parameters of sheep placenta and the birth weight of lambs. Large Anim Rev, 26: 119-126.

Cankaya S, Eker S, Abaci SH. 2019. Comparison of Least Squares, Ridge Regression and Principal Component Approaches in the Presence of Multicollinearity in Regression Analysis. Turkish J Agriculture-Food Sci and Tech, 7(8): 1166-1172. DOI: 10.24925/turjaf.v7i8.1166-1172.2515.

Dwyer CM, Calvert SK, Farish M, Donbavand J, Pickup HE. 2005. Breed, litter and parity effects on placental weight and placentome number and consequences for the neonatal behavior of the lamb. Theriogenology, 63: 1092-1110.

Echternkamp SE: 1993. Relationship between placental development and calf birth weight in beef cattle. Anim Reprod Sci, 32; 1-13.

Eyduran E. 2020. ehaGoF: calculates goodness of fit statistics. R package version 0.1.1. URL: https://CRAN.Rproject.org/package=ehaGoF (erişim tarihi: 17.08.2020).

Hoerl AE, Kennard RW. 1970. Ridge Regression: Biased Estimation for Nonorthogonal Problems. Technometrics, 12 (1): 55-67. DOI: 10.2307/1267351.

Imdad MU, Aslam M. 2018a. Imridge: linear ridge regression with ridge penalty and ridge statistics. URL: https://CRAN.Rproject.org/package=lmridge, $\mathrm{R}$ package version 1.2. (erişim tarihi: 17.08.2020).

Imdad MU, Aslam M. 2018b. liureg: liu regression with liu biasing parameters and statistics. URL: https://CRAN.Rproject.org/package=liureg, R package version 1.1.2. (erişim tarihi: 15.08.2020).

Konyali A, Tölü C, Daş G, Savaş T. 2007. Factors affecting placental traits and relationships of placental traits with neonatal behaviour in goat. Anim Reprod Sci, 97: 394-401.

Kurtulus M. 2001. A study on ridge regression. Master Thesis. Gazi University, Graduate School of Natural and Applied Sciences, p. 42, Ankara.

Kutner MH, Nachtsheim CJ, Neter J, Li W. 2004. Applied linear statistical models. 5th edition, McGraw-Hill/Irwin, New York.

Liu K. 1993. A new class of biased estimate in linear regression. Comm Statist Theory Methods, 22(2): 393-402.

Onder H, Abaci SH. 2015. Path analysis for body measurements on body weight of saanen kids. Kafkas Univ Vet Fak Derg, 21(3): 351-354, DOI: 10.9775/kvfd.2014.12500.

Osgerby JC, Gadd TS, Wathes DC. 2003. The effects of maternal nutrition and body condition on placental and foetal growth in the ewe. Placenta, 24: 236-247. DOI:

10.1053/plac.2002.0902.

Ozyurek S, Turkyilmaz D. 2020. Determination of relationships between placental characteristics and birth weight in Morkaraman sheep. Arch Anim Breed, 63: 39-44. DOI: 10.5194/aab-63-39-2020.

Ozyurek S. 2019. Investigation of the relationship between kids vitality and placental characteristics in Hair goats. Large Anim Rev, 25: 173-177.

R Core Team. 2019. R: A language and environment for statistical computing. R Foundation for Statistical Computing, Vienna, Austria. URL: https://www.R-project.org/.

R Core Team. 2020. R: A language and environment for statistical computing. R Foundation for Statistical Computing, Vienna, Austria. URL: https://www.R-project.org/.

Sarstedt M, Mooi E, 2014. A concise guide to market research. Heidelberg: Springer Texts in Business and Economics. Chapter 7: Regression Analysis; p. 193-233. DOI: 10.1007/978-3-642-53965-7_7, \#Springer-Verlag Berlin Heidelberg.

Sen U, Sirin E, Kuran M. 2013. The effect of maternal nutritional status during mid-gestation on placental characteristics in ewes. Animal Reprod Sci, 137: 31-36.

Sen U, Sirin E, Onder H. 2019. Establishment of effective placental characteristics on birth weight in karayaka sheep breed with path analysis. In: International Congress on Domestic Animal Breeding, Genetics and Husbandry 2019 (ICABGEH-19); 2019 September 11-13; Prague, CZECHIA.

Sen U, Onder H. 2016. Poor placental traits reduce kid birth weight in young Saanen dams at the first parity. Turkish J Vet and Anim Sci, 40: 554-561.

Tatliyer A. 2020. The effects of raising type on performances of some data mining algorithms in lambs. KSU J Agric Nat, 23(3): 772-780. DOI:10.18016/ksutarimdoga.vi.651232.

Tirink C, Onder H, Kurnaz FS. 2019. Comparison of least square method, ridge and liu estimators in saanen goats. In: International Congress on Domestic Animal Breeding Genetics and Husbandry 2019; 2019 September 11-13, Prague, CZECHIA. p. 130.

Tirink C, Abacı SH, Önder H. 2020. Comparison of ridge regression and least squares methods in the presence of multicollinearity for body measurements in saanen kids. J Institute of Sci and Tech, 10(2): 1429-1437, ISSN: 2146-0574, eISSN: 2536-4618.

Topal M, Eyduran E, Yaganoglu AM, Sonmez AY, Keskin S, 2010. Use of ridge and principal component regression analysis methods in multicollinearity. J Agri Fac of Atatürk Univ, 41(1): 53-57. ISSN: 1300-9036.

Uckardes F, Efe E, Narinc D, Aksoy T. 2012. Estimation of the egg albumen index in the Japanese quails with ridge regression method. Akademik Ziraat Derg, 1 (1): 11-20.

Vupa O, Gurunlu Alma 0. 2008. Investigation of multicollinearity problem in small samples included outlier value in linear regression analysis. Selcuk Univ J Sci Fac, 31: 97-107. 